\title{
New treatments for advanced and metastatic colorectal cancer - clinical applications
}

\author{
Stephen Clarke, Associate Professor, Senior Staff Specialist in Medical \\ Oncology, Central Sydney Area Health Service, Sydney
}

\section{SYNOPSIS}

In the last five years several new drugs have become available for the treatment of patients with advanced colorectal cancer. These drugs include raltitrexed, capecitabine, irinotecan and oxaliplatin. They have resulted in improved tumour response rates compared with older treatment regimens using 5-fluorouracil. Combinations of these drugs provide hope for future palliation of this common cancer.

Index words: raltitrexed, capecitabine, irinotecan, oxaliplatin.

(Aust Prescr 2002;25:111-3)

\section{Introduction}

Colorectal cancer affects 1 in 20 Australians with Australia, New Zealand and the United States having the highest incidences of this disease in the world. Approximately $25 \%$ of patients will have advanced disease at presentation and, in spite of locally effective surgery, another $25 \%$ of patients will relapse postoperatively. Large numbers of patients could therefore benefit from effective palliative treatments.

For a long time chemotherapy was not offered to patients with advanced or metastatic colorectal cancer. This was partly because this cancer is commonest in patients over 60 years old who were thought to be at greater risk of toxicity from chemotherapy, and also because the drugs available were not particularly effective. However, there is now evidence that chemotherapy prolongs survival for patients with advanced colorectal cancer compared to best supportive care. In addition, for patients responding to therapy the increment in survival can be more than 18 months.

\section{Standard chemotherapy}

The most commonly used chemotherapy for colorectal cancer has been 5-fluorouracil (5-FU) which has been available since 1957.5-FU has multiple mechanisms of cytotoxicity including the inhibition of thymidylate synthase. The toxicities and efficacy of this drug vary significantly with the mode of administration. Short intravenous injections (bolus schedules) produce unpredictable adverse effects with mucositis, diarrhoea and leucopenia predominating.
The activity of 5-FU is increased by the co-administration of folinic acid which enhances the inhibition of thymidylate synthase. A bolus schedule of 5-FU and folinic acid given for five days in a row and repeated every 28 days (the Mayo Clinic Schedule) has been the most favoured regimen, because of survival advantages in randomised trials compared to 5-FU alone. In the elderly and infirm patient, this regimen is often altered to a weekly injection for six out of eight weeks because of a lower incidence of severe mucositis. However, this regimen has not been shown to be superior to 5-FU alone.

5-FU may also be administered as an intravenous infusion, of varying duration. This improves response rates and tolerance although there is no difference in survival. Bolus schedules of 5 -FU produce antitumour responses in $10-20 \%$ of patients treated while infusional regimens achieve response rates of 20-30\% (based on a greater than $50 \%$ fall in the product of tumour diameters on CT scan). There is a much lower incidence of mucositis and myelosuppression with infusional regimens. The commonest adverse effects are related to redness and peeling of the palms and soles (plantar-palmar erythroderma) and to complications from the central venous catheters required to enable outpatient administration of treatment.

The toxicities of most anticancer treatments are usually maximal 7-14 days after treatment. This is true for both established drugs and the newer anticancer treatments. Patients should be asked to contact their local doctor or oncologist if toxicities occur. Routine monitoring usually consists of a complete assessment of the patient's health, and a full blood count and biochemistry on the day of treatment. Reductions of $20-50 \%$ of the initial dose will be made if severe toxicity has occurred in a previous cycle of treatment.

In the last five years a number of new treatments have become available for patients with advanced colorectal cancer. The effectiveness of these drugs needs to be compared with that of 5-FU (Table 1).

\section{Raltitrexed}

Raltitrexed is a folate analogue which inhibits thymidylate synthase. It is given as a 15 minute intravenous infusion every three weeks. It is subsidised by the Pharmaceutical Benefits Scheme (PBS) for the treatment of patients with advanced or recurrent colorectal cancer. 
Table 1

Summary of phase III trials of new treatments in colorectal cancer

\begin{tabular}{lcll}
\hline $\begin{array}{l}\text { Drug or } \\
\text { combination }\end{array}$ & Response rate & $\begin{array}{l}\text { Median survival time } \\
\text { compared to 5-FU }\end{array}$ & $\begin{array}{l}\text { Impact on overall quality of life } \\
\text { compared with 5-FU }\end{array}$ \\
\hline $\begin{array}{l}\text { Raltitrexed } \\
\text { Capecitabine }\end{array}$ & $15-20 \%$ & Equivalent & $\begin{array}{l}\text { Equivalent } \\
\text { (less mucositis and myelosuppression) }\end{array}$ \\
Irinotecan/5-FU & $20-25 \%$ & Equivalent & $\begin{array}{l}\text { Equivalent } \\
\text { (all toxicities less except hand-foot changes) } \\
\text { Equivalent/better } \\
\text { (less myelosuppression/mucositis) } \\
\text { Exuivalent } \\
\text { (more myelosuppression/neuropathy) }\end{array}$ \\
\hline $5-39 \%$ & Superior (2-3 months) & Equivalent &
\end{tabular}

The principal toxicities associated with raltitrexed are fatigue, myelosuppression and occasionally severe diarrhoea. The combination of neutropenia and severe diarrhoea may be lifethreatening. It should be treated with aggressive resuscitation and intravenous antibiotics active against Gram negative bacteria. Patients at greater risk of severe toxicity in whom dose reduction should be considered include those with impaired renal function, low serum albumin and poor day to day functioning (performance status). However, in such patients the likely benefit from treatment is very low and it may be prudent not to treat these patients with chemotherapy. If a patient experiences the combination of diarrhoea and myelosuppression, even in a mild form, they may experience much worse toxicities with subsequent treatment. Dose reductions may be appropriate in these patients.

In large comparative studies raltitrexed has produced equivalent response and survival rates to bolus schedules of 5-FU and folinic acid. ${ }^{1}$ Patients had a lower incidence of myelosuppression and mucositis. However, a more recent randomised study, which compared raltitrexed to two different infusional forms of 5-FU, found increased toxicity and a lower quality of life in patients given raltitrexed. Although overall survival was similar there was an increase in treatmentrelated deaths (approximately 6\% for patients treated with raltitrexed and less than $1 \%$ for patients on the infusional arms). ${ }^{2}$ These excess deaths are thought to be due to a combination of neutropenia and diarrhoea. While drug-induced deaths have been uncommon in the Australian experience with raltitrexed, these results show that it should be used with care. The future for raltitrexed in colorectal cancer may rest with combinations with other active drugs such as oxaliplatin and irinotecan.

\section{Capecitabine}

Capecitabine is an orally administered 5-FU prodrug. Its final enzymatic activation is mediated by thymidine phosphorylase. This enzyme has a higher concentration in tumours than in normal tissues, so it may have a selective action. Capecitabine was developed to mimic continuous infusions of 5-FU so it is taken twice daily. Initial randomised phase II trials of capecitabine compared intermittent (two weeks on and one week off) with continuous therapy, with or without the addition of folinic acid. The treatment with the best therapeutic index was intermittent capecitabine given without folinic acid. This regimen has been used subsequently.

The principal adverse effect of capecitabine is plantar-palmar erythroderma. If this is mild, treatment requires no adjustment or only transient interruption, but more severe cases require a dose reduction. Toxicities such as nausea and vomiting, mucositis and diarrhoea are uncommon. Severe neutropenia occurs in less than $10 \%$ of patients treated. Capecitabine may cause mild abnormalities of liver function including an elevation in bilirubin. It may also cause a rapid escalation of INR in patients taking warfarin and some patients may need to be changed to other forms of anticoagulation.

Two large international randomised studies comparing capecitabine with the Mayo Clinic Schedule of 5-FU and folinic acid have reported equivalent response and survival data for the two treatment arms. ${ }^{3,4}$ There was markedly less leucopenia, diarrhoea and mucositis in the patients taking capecitabine.

Capecitabine is available on the PBS for patients with advanced or recurrent colorectal cancer. In the future it will probably replace infusional 5-FU in combination with radiotherapy for patients with rectal cancer and will be used as part of combination therapy in advanced disease. Single drug capecitabine will remain as a suitable palliative therapy for elderly and infirm patients with advanced colorectal cancer.

\section{Irinotecan}

Irinotecan acts on topoisomerase to prevent religation of breaks in DNA. It may be given as a weekly intravenous injection for four out of six weeks or at a higher dose every three weeks.

Irinotecan may produce severe toxicity, with delayed onset diarrhoea requiring rehydration occurring in over $30 \%$ of patients. This can be ameliorated with the use of high-dose loperamide, $4 \mathrm{mg}$ initially and $2 \mathrm{mg}$ every two hours at the first sign of severe diarrhoea, continuing until the patient has been free of diarrhoea for 12 hours. If the diarrhoea does not settle, intravenous hydration should be considered and if neutropenia co-exists, start antibiotics active against Gram negative organisms. Less commonly an acute, early 
onset, cholinergic mediated diarrhoea may occur, but this settles with atropine. Other toxicities include neutropenia and alopecia. Patients who have had pelvic radiotherapy have been excluded from trials of irinotecan, because of concerns that this might cause more severe diarrhoea, so these patients should be treated with caution. Irinotecan is probably not a treatment which should be lightly instituted in frail and/or elderly patients.

Irinotecan is currently available on the PBS for patients with advanced and recurrent colorectal cancer who have failed therapy with fluoropyrimidines. ${ }^{5,6}$ There are data to suggest that the combination of irinotecan and 5-FU may be an optimal first-line therapy for patients with advanced colorectal cancer. ${ }^{7,8}$

As a second-line therapy, irinotecan treatment produces significantly improved survival compared to either best supportive care or infusional 5-FU. Approximately $20 \%$ of patients will have evidence of a major tumour response. When used first-line, two randomised studies have shown that the combination of irinotecan and 5-FU produces responses in approximately $50 \%$ of patients and provides a survival advantage of several months compared to patients treated with 5-FU and folinic acid. The median survival for patients with advanced colorectal cancer using combination therapy approaches 18 months.

\section{Oxaliplatin}

Oxaliplatin is a platinum derivative which has activity in colorectal cancer, somewhat surprisingly given that cisplatin is inactive. The toxicities produced by oxaliplatin are also different from those seen with cisplatin. Nausea, vomiting and renal impairment, which are dose limiting with cisplatin, are not such major problems with oxaliplatin. The principal and dose limiting toxicity is a predominantly sensory peripheral neuropathy. Initially these symptoms are transient and associated with cold, but after 4-5 months of treatment the symptoms become constant. Slow improvement occurs after cessation of treatment. In addition to the peripheral neuropathy, a cold-related laryngopharyngeal dysaesthesia may occur which can be alarming to patients. Apart from the neuropathy, the main other toxicity associated with oxaliplatin is mild myelosuppression.

Oxaliplatin has been used in European countries for some years and has recently been made available on the PBS for second-line treatment of patients with colorectal cancer. This is somewhat surprising given that there is a lack of data, especially randomised trials, for this indication. However, there are phase II results which show a $10-20 \%$ response rate for oxaliplatin in patients who have failed 5-FU. Data also show anti-tumour activity in patients whose cancers had previously progressed on 5-FU, suggesting some synergy between oxaliplatin and 5-FU. In first-line use, the combination of 5-FU and oxaliplatin produces response rates of approximately 50\%. However, randomised data have not shown a survival benefit over 5-FU and folinic acid, although the trials comparing these regimens were not powered to detect survival differences.

\section{Conclusion}

The treatment options have increased in the last five years for patients with advanced and metastatic colorectal cancer. The new drugs have resulted in improved response rates without worsening of patient quality of life. There are also suggestions of modest improvements in overall survival. Hopefully these treatments may result in improved palliation of this common condition and lead the way to more effective adjuvant treatments in the future.

E-mail: sclarke@canc.rpa.cs.nsw.gov.au

\section{REFERENCES}

1. Cunningham D. Mature results from three large controlled studies with raltitrexed ('Tomudex') [review]. Br J Cancer 1998;77 Suppl 2:15-21.

2. Maughan TS, James RD, Kerr DJ, Ledermann JA, McArdle C, Seymour MT, et al. Comparison of survival, palliation, and quality of life with three chemotherapy regimens in metastatic colorectal cancer: a multicentre randomised trial. Lancet 2002;359:1555-63.

3. Van Cutsem E, Twelves C, Cassidy J, Allman D, Bajetta E, Boyer M, et al. Oral capecitabine compared with intravenous fluorouracil plus leucovorin in patients with metastatic colorectal cancer: results of a large phase III study. J Clin Oncol 2001;19:4097-106.

4. Hoff PM, Ansari R, Batist G, Cox J, Kocha W, Kuperminc M, et al. Comparison of oral capecitabine versus intravenous fluorouracil plus leucovorin as first-line treatment in 605 patients with metastatic colorectal cancer: results of a randomized phase III study. J Clin Oncol 2001;19:2282-92.

5. Cunningham D, Pyrhonen S, James RD, Punt CJ, Hickish TF, Heikkila R, et al. Randomised trial of irinotecan plus supportive care versus supportive care alone after fluorouracil failure for patients with metastatic colorectal cancer. Lancet 1998;352:1413-8.

6. Rougier P, Van Cutsem E, Bajetta E, Niederle N, Possinger K, Labianca R, et al. Randomised trial of irinotecan versus fluorouracil by continuous infusion after fluorouracil failure in patients with metastatic colorectal cancer [published erratum appears in Lancet 1998;352:1634]. Lancet 1998;352:1407-12.

7. Saltz LB, Cox JV, Blanke C, Rosen LS, Fehrenbacher L, Moore MJ, et al. Irinotecan plus fluorouracil and leucovorin for metastatic colorectal cancer. Irinotecan Study Group. N Engl J Med 2000;343:905-14.

8. Douillard JY, Cunningham D, Roth AD, Navarro M, James RD, Karasek P, et al. Irinotecan combined with fluorouracil compared with fluorouracil alone as first-line treatment for metastatic colorectal cancer: a multicentre randomised trial [published erratum appears in Lancet 2000;355:1372]. Lancet 2000;355:1041-7.

Dr Clarke has acted as a consultant to Sanofi-Synthelabo.

\section{Self-test questions}

The following statements are either true or false (answers on page 123)

3. Diarrhoea can be a life-threatening complication of raltitrexed.

4. Oxaliplatin is more active than cisplatin against colorectal cancer. 